\title{
Les documents et artefacts Molson a l'Université McGill
}

par

Alfred Dubuc

L'auteur propose un court inventaire des documents et des artefacts de la famille Molson qui se trouvent dans les diverses bibliothèques, depôts d'archives et musées de l'Université McGill. Il distingue trois catégories de documents: $1^{\mathbf{e}}$ les documents de l'Université McGill concernant les Molson, leurs nombreuses donations et la participation de plusieurs membres de la famille au bureau des gouverneurs de l'Université; ces documents se trouvent aux Archives de McGill; $2^{\mathbf{e}}$ des documents et des artefacts provenant de la famille Molson et conservés au musée Redpath, au musée McCord d'histoire canadienne et au département des livres rares et des collections spéciales situé dans la bibliothèque McLennan; $3^{\mathbf{e}}$ des documents concernant les Molson, donnés à l'Université Mc'Gill par d'autres personnes; il s'agit principalement de photographies conservées dans la collection Notman du musée McCord. L'auteur décrit, en outre, l'origine du nom Molson donné à trois édifices du campus.

This article gives a brief listing of Molson family documents which are kept in McGill University libraries, archives and museums. Three categories are identified: 1) documents in the archives related to numerous donations to the university and to the participation by Molson family members in the administration of the university. 2) Molson documents and artefacts kept in the McGill museums and libraries (Redpath Museum, McCord Museum and McLennan Library). 3) Documents concerning the Molson family, mainly photographs in the Nottman Collection (McCord Museum). This article also briefly describes three buildings on McGill campus named after Molson family members.

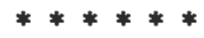

Toute communauté d'hommes d'affaires se dote d'institutions sociales, culturelles, religieuses et de bienfaisance ayant pour but de répondre à ses responsabilités envers la collectivité, de satisfaire le besoin d'étendre les ramifications de son influence sur la société et de reproduire, pour sa postérité, les conditions d'exercice de son activité économique et de son pouvoir. Celle de Montréal ${ }^{1}$ n'échappe pas à cette rêgle et l'Université McGill, depuis le fameux testament de James McGill, décédé en 1813, pourvoyant au don du domaine Burnside à la Royal Institution for the Advancement of Learning, fut un des bénéficiaires les plus grassement dotés de cette philanthropie. ${ }^{2}$ Certes, les hommes d'affaires ne sont pas tous également généreux et peu le furent autant que les Molson envers McGill. ${ }^{3}$ Un dicton l'exprime à sa façon: "C'est la bière Molson et les cigarettes Macdonald qui font vivre McGill"; comme bien des dictons, toutefois, celui-ci déforme légèrement la réalité car ce fut tout autant à titre de banquiers que les Molson exprimèrent leur générosité, depuis l'époque où les trois frères, John l'Aîné, William et Thomas, à l'occasion de la campagne de souscription, lancée par le principal Sir John William Dawson, ${ }^{4}$ en 1856 , pour la première fondation de McGill, offraient $£ 5,000$ (\$20,000) sur un total de $£ 15,000$ $(\$ 60,000)$ souscrits par 50 personnes; l'annee précédente, leur banque, 
Molson's Bank, fondée en 1853 suivant les dispositions de la loi des banques privées, était devenue une banque à charte. ${ }^{5}$

On trouve à l'Université McGill des traces nombreuses de cette sollicitude souvent répétée. J'appelle "documents Molson", pour les fins de cet article, trois groupes de matériaux: le premier est constitué des édifices du campus qui portent le nom Molson et qui témoignent matériellement, aux yeux de tous, de certaines donations majeures des membres de la famille; le deuxième groupe est conservé aux archives de McGill et exprime la participation des Molson au financement et à la vie de l'Université; le troisième, enfin, est constitué de documents et d'artefacts issus de la famille Molson et conservés dans trois depôts de l'université McGill: le musée Redpath, le musée McCord d'histoire canadienne et la section "Manuscripts" du fonds des "Rare Books" dans la bibliothèque McLennan. ${ }^{6}$

\section{Les pavillons "Molson" sur le campus}

La présence des Molson à McGill est évidente, elle est matérielle: 1. le William Molson Hall à l'extrémité occidentale du pavillon des Arts, inauguré le 10 octobre 1862, en présence du Gouverneur général de la Province unie du Canada, Sir Charles Stanley Monk, et dont l'étage inférieur servit de bibliothèque universitaire durant 30 ans, jusqu'en 1893, quand elle déménagea dans le nouvel édifice de la bibliothèque Redpath; 2. le Percival Molson Memorial Stadium, construit au flanc du Mont Royal et inauguré à l'automne 1919, dont le nom commémore celui qui, tué au combat le 5 juillet 1917, à Avion, près de Arras, en France, avait légué la somme de $\$ 75,000$ pour l'achèvement de ce projet dont il s'était fait le champion depuis déjà bien avant la guerre; 3. la Molson Residence, elle aussi sur le flanc de la montagne, au-dessus du stade, nommée à la suite d'un don évalué à $\$ 600,000$, en 1958, de la Fondation Molson; cette fondation avait été établie la même année par les deux fils du colonel Herbert Molson, le sénateur Hartland de Montarville et Thomas Henry Pentland; elle s'est étendue depuis cette époque et s'appelle aujourd'hui The Molson Family Foundation.

Ainsi la présence des Molson s'exprime matériellement à tous les niveaux de la topographie du campus.

\section{Les archives de McGill}

La présence physique des Molson s'est manifestée parfois, de façon plus au moins évidente aux yeux de ceux qui oeuvraient à McGill, par la participation au Bureau des Gouverneurs de l'un ou de l'autre des membres de la famille, génération après génération, depuis l'époque où William, président de la Molson's Bank, y avait accédé le premier. Mais l'on pourrait remonter encore plus loin dans le temps, jusqu'en 1819, lorsque, à la fondation de la Montreal General Hospital, ${ }^{7}$ John Molson l'Ancien souscrivit généreusement, avec ses trois fils John l'Aîné, Thomas et William, à la construction du nouvel edifice, inauguré en 1824, participa au bureau des gouverneurs et contribua à établir la Montreal Medical Institution qui devint, en 1829, la Faculté de médecine du McGill College. Un demi-siècle plus tard, son petit fils, John Henry Robinson, à qui il avait légué la brasserie, lui-même président de la Molson's Bank à partir de 1889, s'intéressera particuliérement 
à cette faculté en défrayant le coût d'érection d'un amphithéatre de démonstration en annexe de l'ancien pavillon de médecine et, en 1895, en souscrivant $\$ 62,000$ pour la construction d'une nouvelle aile de l'édifice de la même faculté, sans compter le don du terrain, le long de la rue McTavish, destiné au bâtiment de la bibliothèque Redpath, construit en 1893, et sa participation de $\$ 50,000$, en 1894 , au fonds de pension des employés de l'université McGill.

On retrouve également les Molson dans le débat qui précéda l'admission des femmes à McGill en 1884; à l'automne de 1871, quelques dames s'étaient réunies à la résidence de Anne Molson (1824-1899) (Figure 8), fille de William et épouse de John Molson $\mathrm{III}^{8}$ et avaient fondé la Ladies Educational Association of Montreal, dans le but de recevoir l'enseignement d'un groupe de professeurs de McGill, consentant à donner des cours à celles à qui l'Université ne voulait conférer de diplômes; les cours commencèrent en octobre, le premier professeur, dit-on, étant le principal Dawson, lui-même un physicien. Cet enseignement se poursuivit à l'extérieur de l'Université pendant treize ans.

Ce ne sont là que quelques exemples, tirés d'une période que je connais davantage par les recherches que j'ai effectuées sur l'histoire des premiers Molson. ${ }^{9}$ Les archives de McGill foisonnent de documents de toutes natures témoignant de cette activité des membres d'une famille qui, génération après génération, à travers le XXe siecle tout autant qu'à travers le XIXe, ont pourvu McGill de plusieurs de ses richesses et, à titre de gouverneurs, ont contribué à orienter son évolution.

\section{Le musée Redpath}

Le musée a reçu de quelques membres de la famille Molson des artefacts qui ressemblent davantage à des souvenirs de voyages qu'à des objets ayant quelque valeur ethnologique. Toutefois, par leur ancienneté et par leur origine, certains d'entre eux présentent un certain intérêt. Ils sont rassemblés en cinq groupes, selon les noms des donateurs:

1. Velina Pauline Nesmith, originaire de Portland, Oregon, deuxième épouse de William Markland Molson (1833-1873).

2. Mabel Molson (1879-1973), fille de John Thomas et soeur du colonel Herbert Molson.

3. John Henry Robinson Molson (1826-1897), fils aîné de Thomas Molson.

4. John Thomas Molson (1837-1910), fils benjamin de Thomas Molson.

5. Mary A. Kingman, épouse de Walter Molson (1883-1953), fils de John Thomas. 


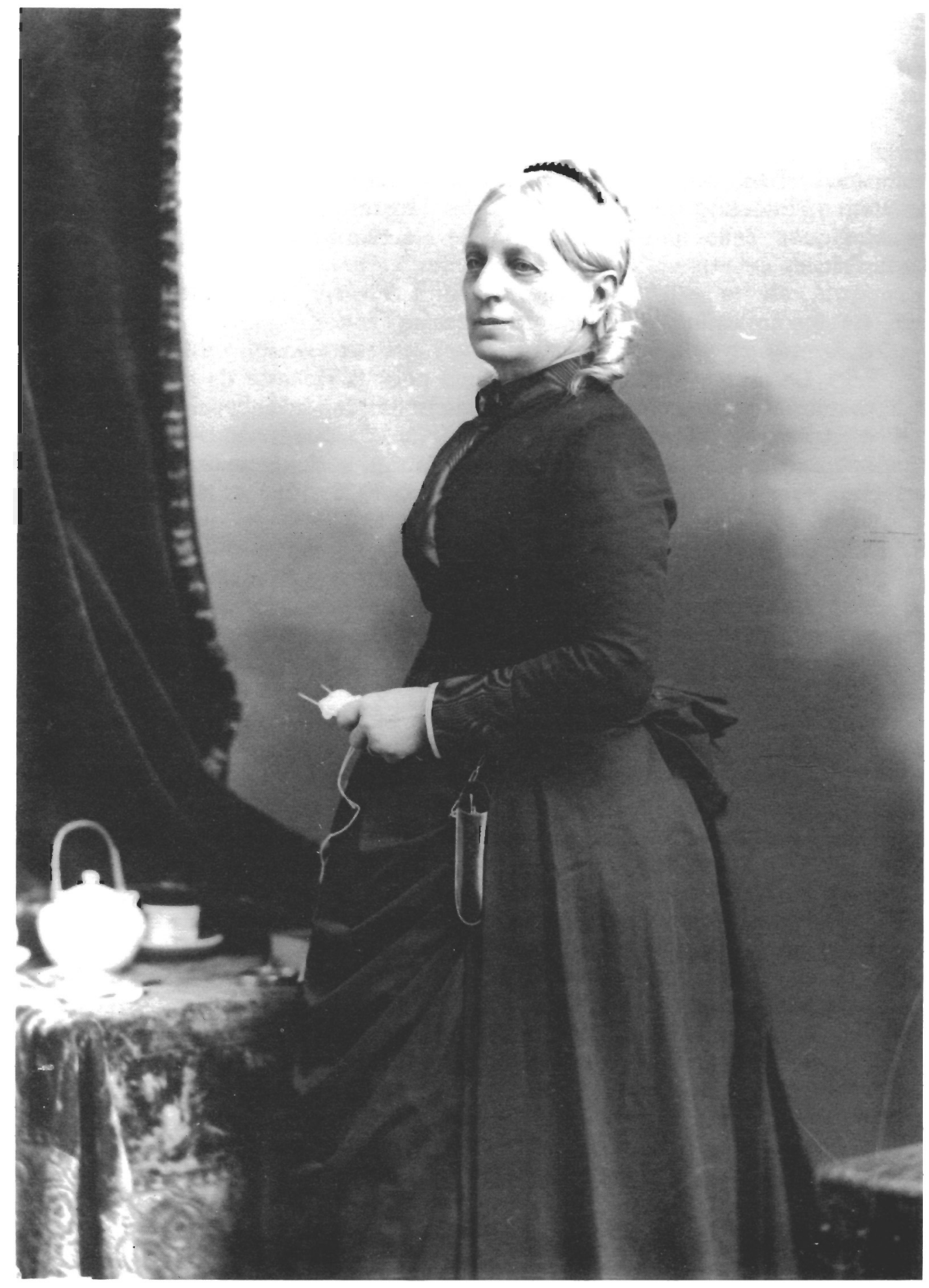

Fig. 8. Mme. John Molson (1824-1899). (Courtesy of Notman Photographic Archives) 


\section{Le musée McCord d'histoire canadienne}

Ce musée est un lieu polyvalent de conservation et d'exposition où l'on retrouve des collections de plusieurs catégories et un centre d'archives historiques. En ce qui concerne l'histoire des Molson, quatre collections suscitent l'intérêt: la collection des costumes anciens, la collection ethnologique, celle des photographies Notman et, enfin, le fonds de manuscrits.

\subsection{La collection de costumes anciens}

Le musée McCord est reconnue pour la richesse de sa collection de costumes anciens; plusieurs expositions ont rendu celle-ci célèbre et ont contribué à diffuser le nom de McCord. Madame Beatrice Stewart, épouse de Thomas Henry Pentland Molson, a donné au musée, en 1972, cinq robes de bal datant des années 1927, 1928, 1950 et 1954.

\subsection{La collection ethnologique}

En ce qui concerne les Molson, cette collection comporte deux séries d'artefacts: celle de la vannerie de Madame Mabel Molson et l'ensemble désigné sous le nom de "Molson collection".

\subsubsection{La collection de vannerie de Madame Mabel Molson}

En 1928, Madame Mabel Molson, (1879-1973) fille de John Thomas et de Jenny B. Butler, soeur du colonel Herbert et de Percival Molson, fit don au musée McCord d'une centaine d'artefacts de vannerie provenant des Inuits et des Amérindiens de la côte du Pacifique, depuis l'archipel des Aléoutiennes jusqu'à l'Etat de Washington, des régions du fleuve Mackenzie et du Grand lac de l'Esclave; la collection contient surtout des paniers utilisés dans la vie courante ou dans les rites de funérailles; elle contient aussi quelques chapeaux de vannerie. A cette collection de Mabel Molson, le musée McCord a ajouté quelques spécimens d'autres provenances. Le catalogue de la collection présente, en première partie, une description ethnographique des techniques de la vannerie, fort utile pour l'appréciation de ces artefacts.

\subsubsection{La collection d'artefacts Molson}

Plus de dix membres de la famille Molson ont donné au musée, durant une période s'étendant de 1924 à nos jours, une quantité considérable d'artefacts présentant un intérêt ethnologique certain; des photographies, des dessins, des peintures, des vêtements, des poupées, des objets de la vie courante, des bijoux, de la vaisselle, des meubles, etc.. L'ensemble le plus considérable et, de loin, le plus intéressant, est celui que Madame Mabel Molson a donné, en plus de sa collection de vannerie.

\subsection{La collection de photographies Notman}

Le musée McCord détient une collection inestimable de 
photographies des XIXe et XXe siècles, dont le fonds principal est constitué des photographies prises par le photographe William (le père), ${ }^{10}$ décédé en 1891, son fils William McFarland, décédé en 1913 et le benjamin des fils, Charles F. Notman, qui vendit l'entreprise en 1935 à la firme Associated Screen News. Le fonds Notman contient plus de 400,000 photographies (et près de 200,000 négatifs) prises entre 1858 et 1935; il fut donné au musée McCord en 1956 grâce à une donation conjointe de la Maxwell Cummings Family Foundation, de M. Paul Nathanson, de la maison Empire Universal Film et de Maclean's Magazine. A ce fonds principal, le musée McCord a ajouté des photographies données par des familles et des individus de Montréal.

En ce qui concerne les Molson, on trouve des photographies dans le fonds Notman et dans d'autres fonds donnés, l'un par Madame Thomas Henry Pentland Molson (Celia Frances Cantlie) et l'autre par Madame Hartland de M. Molson (Helen K. S. Hogg). Ces photographies peuvent être regroupées selon ce qu'elles représentent: de très nombreux portraits (plus d'une centaine pour la seule période 1858-1874) dans le fonds Notman; les demeures de plusieurs membres de la famille (y compris Belmont Hall, acquise par John Molson l'Ancien en 1825, au carrefour des rues Sherbrooke et St-Laurent); finalement, des photographies d'intérêts variés, représentant des familles, des lieux et des événements divers.

Les photographies ajoutent beaucoup à la qualité de la connaissance que nous pouvons avoir de l'histoire des Molson.

\subsection{Le fonds de manuscrits de McCord}

Plusieurs personnes ont donné des documents personnels au musée McCord. Certains membres de la famille Molson ont fait de même. Les documents Molson sont gardés dans trois boites, les deux premières portant la cote commune M 21228 et contenant 17 chemises, les chemises I à X dans la première, XI à XVII dans la seconde; la troisième boite contient des document variés portant chacun une cote séparée; ce sont en majorité des carnets de notes. Les documents les plus nombreux concernent John Thomas Molson (1837-1910), tant dans le premier groupe que dans le deuxième. D'autres documents me sont apparus particulièrement précieux parce qu'ils complètent une série de documents semblables contenus dans le fonds Molson des Archives nationales du Canada: ce sont les carnets de notes de Thomas Molson (1791-1863) (Figure 9). Comme son père, John l'Ancien (1763-1836) et comme son fils John Thomas, mais de façon plus continue et avec davantage de persévérance, Thomas gardait sur lui des petits carnets dans lesquels il inscrivait des informations de toute nature: parmi les plus intéressantes, les unes concernent sa biographie, d'autres, ses entreprises, d'autres, encore - peut-être les plus précieuses - décrivent les techniques de la brasserie, de la distillerie, de la meunerie, du moteur à vapeur, de la navigation, etc.. Les Archives nationales du Canada conservent 12 de ces carnets; le musée McCord en possède 8 , portant sur les années 1815$16,1820,1823-24,1828-32,1835-36,1856,1858$ et 1861 (le catalogue en attribue quelques-uns à John Thomas, mais l'écriture ne peut tromper, ils sont bien de Thomas). 


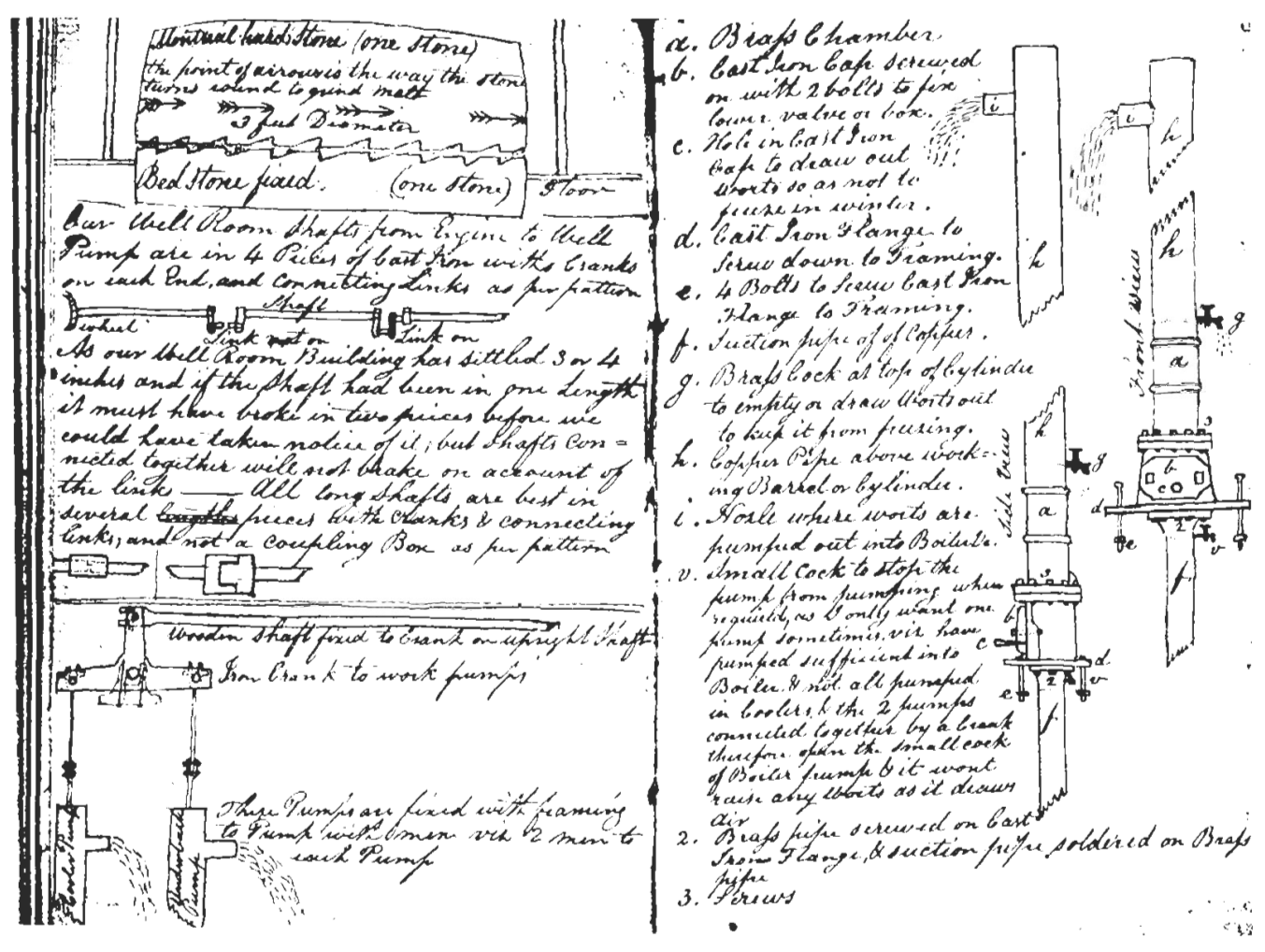

Fig. 9. Carnet de notes de Thomas Molson (1791-1863), Fonds Molson, M19113. (Courtesy of McCord Museum of Canadian History) 


\section{Les manuscrits conservés à la bibliothèque McLennan}

Selon Mrs. Mabel Good, l'ancienne archiviste des Molson, avant que ceux-ci ne fissent don de leur fonds aux Archives nationales du Canada, c'est le Colonel Herbert Molson (1875-1938) qui aurait donné à l'Université McGill une collection de plus de 80 volumes provenant des navires et des entreprises de navigation des Molson depuis 1819 jusqu'en 1838. John Molson l'Ancien avait lancé à Montréal, en 1809 , le premier bateau à vapeur de l'histoire du Canada, le Accomodation, à peine deux ans après que John Fulton eut lancé le Claremont sur l'Hudson. ${ }^{11}$ Il détiendra pendant quelques années le monopole de la navigation à vapeur sur le St. Laurent lançant quatre bateaux de 1812 à 1816 , puis affrontera, durant les années suivantes, la compétition de concurrents de plus en plus nombreux; en 1822, il formera, avec ses trois fils et les propriétaires de trois bateaux concurrents, la $S t$. Lawrence Steamboat Company, dans laquelle les quatre Molson détiendront 26 de 44 actions et de la gestion de laquelle l'entreprise familiale, la John Molson \& Sons, prendra la responsabilité. Dorénavant, la concurrence se fera à deux, la Saint-Lawrence Steam Tow Boat Company (connue aussi sous le nom de Montreal Tow Boat Company qui prendra plus tard le nom de Montreal and Quebec Steamboat Company) des frères Torrance ${ }^{12}$ ayant relevé le gant; mais cette concurrence sera atténuée, les deux compagnies passant des ententes de cartel comprenant même, parfois, la propriété en commun de certains bateaux.

Il n'est pas nécessaire de décrire le contenu de chaque volume pour convaincre de l'importance de cette documentation; on y trouve de l'information sur les passagers et les cargaisons de 15 navires et de 2 barges ayant navigué entre Montréal et Québec de 1819 à 1838. Tous ces volumes n'ont pas le même contenu, et l'information n'y est pas d'égale qualité, mais l'on peut trouver, outre le nom des vaisseaux, le nom des membres des équipages, la quantité de combustible (bois/charbon), le nombre de descentes et de remontées du fleuve durant la saison de navigation (de mai à novembre); la liste de passagers des cabines et du pont, le fret maritime destiné à Montréal et à Québec, mais aussi aux ports d'escales le long des deux rives du fleuve (Sorel, Trois-Rivières, etc.) et sur le Richelieu (Chambly); le nom et l'adresse des expéditeurs et des consignataires des marchandises. D'autre volumes portent sur des sujets plus particuliers qui soulèvent beaucoup d'intérêt pour les chercheurs, comme les cahiers des salaires du vapeur Québec pour l'année 1826 et ceux de l'ensemble des vapeurs et des barges pour les années 1832-1835.

Ici, encore, comme au musée McCord, on trouve des carnets de notes de Thomas Molson (attribués à son frère William), trois en tout. Ajoutés à ceux du musée McCord, l'ensemble des 11 carnets conservés à McGill forme donc près de la moitié des 23 carnets de Thomas Molson qui sont parvenus jusqu'à nous. L'intérêt pour ces carnets ne peut sans doute pas être partagé par tous, mais pour celui qui s'est attardé longuement à la biographie de cet homme ${ }^{13}$, cette documentation présente beaucoup de richesses.

Voilà une description sommaire des documents et des artefacts que l'on 
trouve dans les divers musées et dépôts d'archives de l'Université McGill et qui contribuent à notre connaissance de la famille Molson. Certes, il s'agit d'une documentation accessoire, car le fonds principal des archives Molson a été donné aux Archives nationales du Canada. Tout accessoire qu'il soit, cependant, il ajoute des éléments de connaissance parfois indispensables, particulièrement en ce qui concerne l'activité incessante de cette famille en regard de ses responsabilités envers la grande maison d'enseignement qu'elle a soutenue depuis les tout débuts de son existence.

\section{$\underline{\text { Notes }}$}

1. Sur la communauté des hommes d'affaires de Montréal au XIX ${ }^{\mathbf{e}}$ siècle: Creighton, Donald G., The Empire of the St. Lawrence, Toronto, Macmillan, 1956; Ryerson, Stanley B., Le Capitalisme et la Confédération; aux sources du conflit Canada-Québec (1760-1873), traduction de André D'Allemagne, Montreal, Parti-Pris, 1972; Tulchinsky, Gerald, The River Barons: Montreal Businessmen and the Growth of Industry and Transportation, 1837-1853, Toronto, University of Toronto Press, 1977. Chacun de ces ouvrages fait une place importante aux Molson.

2. Sur l'histoire de l'Université McGill: Frost, Stanley B., McGill University, for the Advancement of Learning, 2 vol., McGill-Queen's University Press, 1980, 1984; sur James McGill: Cooper, John Irwin, "McGill, James," Dictionnaire biographique du Canada, V, 579-583; sur les difficultés concernant l'application du testament de James McGill: Frost, Stanley B., "Desrivieres (Trottier Desrivières) François," Dictionnaire biographique $d u$ Canada, VI, 223-225.

3. Sur l'histoire des Molson: Sandwell, Bernard K., The Molson Family, Montréal, édition privée, 1933; Denison, Merrill, $A u$ Pied du Courant, l'histoire Molson, traduction de Alain Grandbois, Montréal, Beauchemin, 1955; Woods, Shirley E., Jr., La Saga des Molson, 1763-1983, traduction de MarieCatherine Laduré, Montréal, Editions de l'Homme, 1983; Dubuc, Alfred, Les premiers Molson, Montréal, Boréal, à paraître.

4. Eakins, Peter \& Jean, "Dawson, John William," Dictionnaire biographique du Canada, XII, à paraître.

5. Shortt, Adam, "Currency and Banking, 1840-1867," in Neufeld, Edward Peter, ed., Money and Banking in Canada; Historical Documents and Commentary, Carleton Library Series 17, Toronto, McClelland \& Stewart, 1964, 132-148; Breckenridge, R. M., The Canadian Banking System, 18171890, Toronto, 1894.

6. Je tiens à souligner l'aide précieuse que les personnes suivantes m'ont apportée pour la préparation de ce texte: Madame Phebe Chartrand, des Archives de McGill, Madame Barbara Lawson du musée Redpath; au musée McCord, Madame Pamela Miller, de la section "Manuscripts", Monsieur Conrad Graham de la collection d'ethnologie, Madame Jacqueline Beaudoin Ross, de la section des costumes anciens, et Madame Nora Hague de la collection Notman; M. Richard Virr, de la collection de manuscrits du fonds des "Rare Books"; je 
les en remercie vivement.

7. MacDermot, H. E., A History of the Montreal General Hospital, Montréal, 1950; la participation des Molson à la fondation de l'hôpital est mentionnée aux pages IV, 2, 4, 12, 34, 41, 42, 110; sur la participation des Molson aux premières étapes de l'histoire de Montréal au XIX ${ }^{\mathbf{e}}$ siècle: Doige, Thomas, An Alphabetical List of Merchants, Traders and Housekeepers Residing in Montreal, Montréal, 1819, $2^{\mathrm{e}}$ edit. 1820, 20-1, 23, 26, 30, 108; Berthelot, Hector, Montréal, Le bon vieux temps, compilé, revu et corrigé par E. Z. Massicotte, Montréal, Beauchemin, 1916, partie I, 23-4, 25; partie II, 11, 18, 53; Atherton, W. H., Montreal, 1534-1914, 3 vol., Montréal, 1914, v. II, 138, 271, 275-9, 283, 435, 527, 556, 575-6, 607-8; Borthwick, J., History and Biographical Gazetteer for Montreal to the Year 1892, Montreal, Lovell, 1892, Part I, 34-5, 37-8, 44, 50-1, 53; Bosworth, Newton, Hochelaga Depicta..., Montréal, William Greig, 1839, 100, 109, 124, 127, 152-3; Sandham, Alf red, Ville-Marie or Sketches of Montreal Past and Present, Montréal, George Bishop, 1870, 91-2, 93.

8. Bradbury, Bettina, "Molson, Anne,", Dictionnaire biographique du Canada, XII, à paraître.

9. Dubuc, Alfred, "Molson, John (l'Ancien)," Dictionnaire biographique du Canada, VII, à paraître; "Molson, John (l'Aîné)", (avec Robert Tremblay), ibid., VIII, 698-703; "Molson, Thomas", ibid., IX, 615-617; "Molson, William", ibid., X, 567-576.

10. Triggs, Stanley G., "Notman, William," Dictionnaire biographique $d u$ Canada, XII, à paraître.

11. Wilson, G. H., "The Application of Steam to the St-Lawrence Valley Navigation, 1809-1840," thèse M. A. (Histoire), Université McGill, 1961; Flexner, James T., Steamboats Come True; American Inventors in Action, New York, Viking, 1944.

12. Armstrong, Frederick H., "Torrance, John," Dictionnaire biographique du Canada, IX, 875-876; "Torrance, David," ibid., X, 747-749.

13. Dubuc, Alfred, "Thomas Molson, entrepreneur canadien, 1791-1863" Doctorat ès Lettres (Histoire), Université de Paris (Sorbonne), 1969. 